\title{
New pole contribution to \\ transverse-momentum-weighted single-transverse \\ spin asymmetry in semi-inclusive deep inelastic scattering
}

\section{Shinsuke Yoshida*}

Key Laboratory of Quark and Lepton Physics (MOE) and Institute of Particle Physics, Central China Normal University, Wuhan 430079, China

E-mail: shinyoshida85@gmail.com

\begin{abstract}
We discuss a new hard pole contribution to the transverse-momentum-weighted single-transverse spin asymmetry in semi-inclusive deep inelastic scattering. We perform a complete next-toleading order calculation of the $P_{h \perp}$-weighted cross section and show that the new hard pole contribution is required in order to obtain the complete evolution equation for the twist-3 QiuSterman function.
\end{abstract}

XXIV International Workshop on Deep-Inelastic Scattering and Related Subjects

11-15 April, 2016

DESY Hamburg, Germany

\footnotetext{
* Speaker.
} 


\section{Introduction}

In a perturbative QCD calculation, higher-order corrections bring the logarithmic energyscale dependence of nonperturbative function which is described by the scale evolution equation. Systematic treatment of the scale dependence of the twist-3 functions is important for a quantitative description of the single-transverse spin asymmetry(SSA). The twist-3 distribution effect of the transversely polarized proton is embodied as the so-called Qiu-Sterman (QS) function $G_{F}\left(x_{1}, x_{2}\right)\left(T_{F}\left(x_{1}, x_{2}\right)\right)$ in the spin-dependent cross section formula. The scale evolution equation of the QS function was discussed by using several different approaches so far $[1,2,3,4,5,6,7,8]$. One of the approaches is the next-to-leading-order (NLO) calculation of the transverse momentum $P_{h \perp}$-weighted cross section. We present a complete NLO cross section for the twist- $3 P_{h}$-weighted cross section for SSA in semi-inclusive deep inelastic scattering.

We consider the SSA for light-hadron production in SIDIS,

$$
e(\ell)+p\left(p, S_{\perp}\right) \rightarrow e\left(\ell^{\prime}\right)+h\left(P_{h}\right)+X .
$$

Within the collinear factorization framework, the SSA can be described by the twist-3 effects. In this process, the SSA receives two types of twist-3 contributions, the distribution effect of the transversely polarized proton and the fragmentation effect of the light-hadron. We focus on the former contribution in this paper to derive the evolution equation of $G_{F}(x, x)$. In the case of SIDIS, the cross section formula can be expressed in terms of the following Lorentz invariant variables,

$$
S_{e p}=(p+\ell)^{2}, \quad Q^{2}=-q^{2}, \quad x_{B}=\frac{Q^{2}}{2 p \cdot q}, \quad z_{h}=\frac{p \cdot P_{h}}{p \cdot q},
$$

where $q=\left(\ell-\ell^{\prime}\right)$ is the momentum of the virtual photon. In this paper, we discuss the NLO $P_{h \perp}$-weighted polarized cross section defined as

$$
\frac{d^{4}\left\langle P_{h \perp} \Delta \sigma\right\rangle}{d x_{B} d Q^{2} d z_{h} d \phi} \equiv \int d^{2} P_{h \perp} \varepsilon^{\alpha \beta+-} S_{\perp \alpha} P_{h \perp \beta}\left(\frac{d^{6} \Delta \sigma}{d x_{B} d Q^{2} d z_{h} d P_{h \perp}^{2} d \phi d \chi}\right) .
$$

\section{Leading-order cross section and next-to-leading-order virtual correction cotribution}
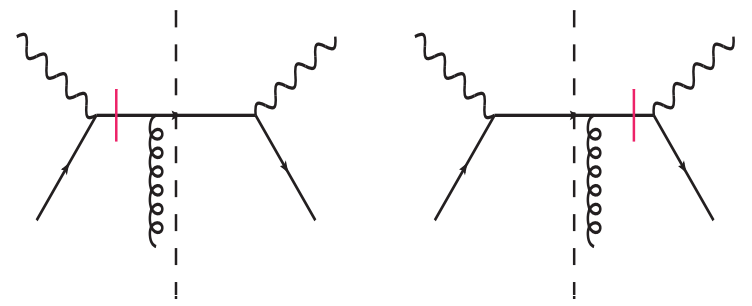

Figure 1: Leading order diagram for the $P_{h \perp}$-weighted cross section. The red barred propagators give pole contributions.

We discuss a leading-order(LO) $P_{h \perp}$-weighted cross section. LO diagrams are shown in Fig. 1. In the collinear factorization approach, the SSA requires a complex phase given by a pole of 
an internal propagator. The red barred propagators in Fig. 1 give pole contributions. The LO cross section can be easily calculated as

$$
\frac{d^{4}\left\langle P_{h \perp} \Delta \sigma\right\rangle^{\mathrm{LO}}}{d x_{B} d Q^{2} d z_{h} d \phi}=-\frac{z_{h} \pi M_{N} \alpha_{e m}^{2}}{4 x_{B}^{2} S_{e p}^{2} Q^{2}} \sum_{q} e_{q}^{2} G^{q}\left(x_{B}, x_{B}\right) D^{q}\left(z_{h}\right),
$$

where $M_{N}$ is the nucleon mass and $\alpha_{e m}$ is the QED coupling constant. The next-to-leadingorder(NLO) virtual correction contribution is given by one-loop correction to the LO diagrams. This has been calculated in [8] as

$$
\begin{aligned}
\frac{d^{4}\left\langle P_{h \perp} \Delta \sigma\right\rangle^{\text {virtual }}}{d x_{B} d Q^{2} d z_{h} d \phi}= & -\frac{z_{h} \pi M_{N} \alpha_{e m}^{2}}{4 x_{B}^{2} S_{e p}^{2} Q^{2}} \frac{\alpha_{s}}{2 \pi} \sum_{q} e_{q}^{2} G^{q}\left(x_{B}, x_{B}\right) D^{q}\left(z_{h}\right) \\
& \times\left[C_{F}\left(\frac{4 \pi \mu^{2}}{Q^{2}}\right)^{\varepsilon} \frac{1}{\Gamma(1-\varepsilon)}\left(-\frac{2}{\varepsilon^{2}}-\frac{3}{\varepsilon}\right)\right]+\cdots
\end{aligned}
$$

Here we adopted the dimensional regularization scheme and $\varepsilon=2-D / 2$. We neglect $O(1)$ contributions throughout.

\section{Next-leading-order real emission contribution}
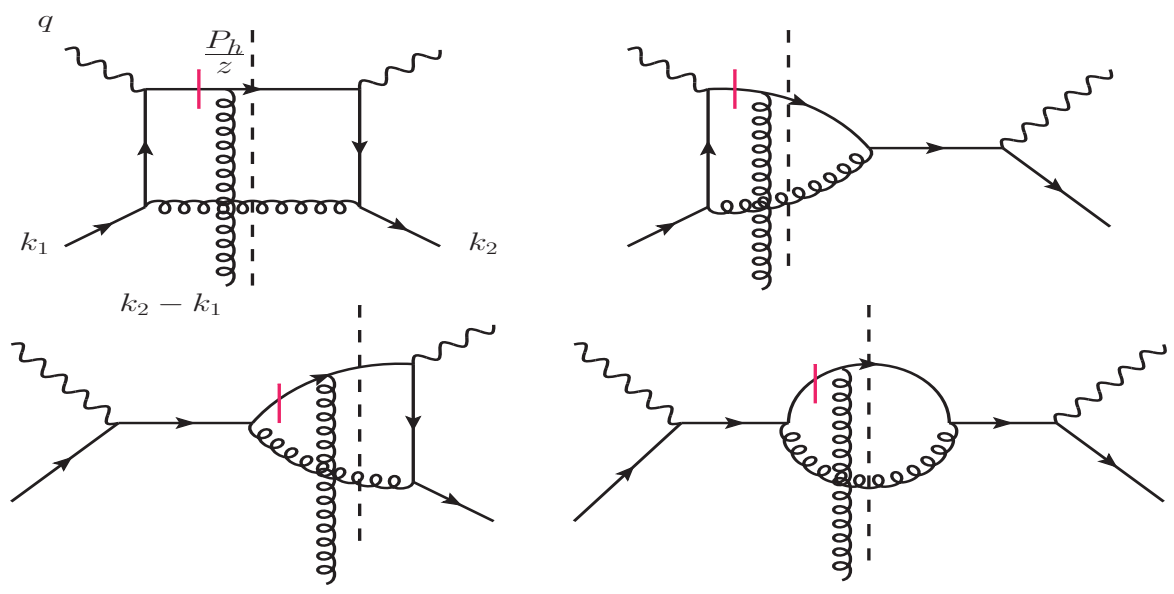

Figure 2: Diagrammatic description for SGP contribution.

In this section, we discuss NLO real emission contributions. In SIDIS case, the pole contributions can be classified into four types as soft-gluon-pole(SGP), soft-fermion-pole(SFP), hardpole(HP) and another hard-pole(HP2) [9, 10]. It was found in [11] that the SFP contribution is completely cancelled in the SIDIS case. We discuss other 3 pole contributions below.

First we discuss the SGP contribution. This is given by the diagrams in Fig. 2. The SGP contribution has been calculated in [8] as

$$
\frac{d^{4}\left\langle P_{h \perp} \Delta \sigma\right\rangle^{\mathrm{SGP}}}{d x_{B} d Q^{2} d z_{h} d \phi}
$$




$$
\begin{aligned}
= & -\frac{\pi M_{N} \alpha_{e m}^{2}}{4 x_{B}^{2} S_{e p}^{2} Q^{2}} \frac{\alpha_{s}}{2 \pi}\left(\frac{4 \pi \mu^{2}}{Q^{2}}\right)^{\varepsilon} \frac{1}{\Gamma(1-\varepsilon)} \int d z D(z) \int \frac{d x}{x} G_{F}(x, x) \\
& \times \frac{1}{2 N}\left[-\frac{2}{\varepsilon^{2}} \delta(1-\hat{x}) \delta(1-\hat{z})+\frac{1}{\varepsilon} \frac{1+\hat{z}^{2}}{(1-\hat{z})_{+}} \delta(1-\hat{x})-\frac{1}{\varepsilon} \frac{2 \hat{x}^{3}-3 \hat{x}^{2}-1}{(1-\hat{x})_{+}} \delta(1-\hat{z})\right]+\cdots,(3.1
\end{aligned}
$$

where $\alpha_{s}$ is the QCD coupling constant, $\hat{x}=x_{B} / x$ and $\hat{z}=z_{h} / z$.
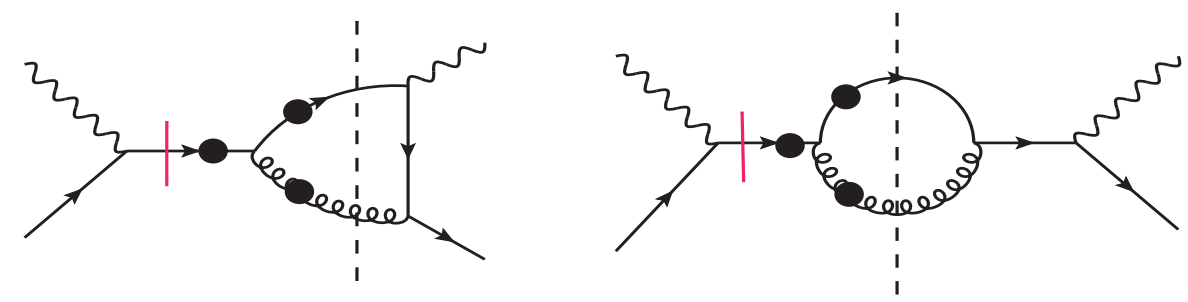

Figure 3: Diagrammatic description for HP contribution.

Next we discuss the HP contribution shown in Fig. 3. This contribution can be calculated as

$$
\begin{aligned}
& \frac{d^{4}\left\langle P_{h \perp} \Delta \sigma\right\rangle^{\mathrm{HP}}}{d x_{B} d Q^{2} d z_{h} d \phi} \\
= & -\frac{\pi M_{N} \alpha_{e m}^{2}}{4 x_{B}^{2} S_{e p}^{2} Q^{2}} \frac{\alpha_{s}}{2 \pi}\left(\frac{4 \pi \mu^{2}}{Q^{2}}\right)^{\varepsilon} \frac{1}{\Gamma(1-\varepsilon)} \int d z D(z) \int \frac{d x}{x}\left(\hat{z} C_{F}+\frac{1}{2 N}\right) \\
& \times\left\{G _ { F } ( x , x _ { B } ) \left[\frac{2}{\varepsilon^{2}} \delta(1-\hat{x}) \delta(1-\hat{z})+\frac{1}{\varepsilon}\left(2 \delta(1-\hat{x}) \delta(1-\hat{z})-\frac{1+\hat{z}^{2}}{(1-\hat{z})_{+}} \delta(1-\hat{x})\right.\right.\right. \\
& \left.\left.\left.-\frac{1+\hat{x}}{(1-\hat{x})_{+}} \delta(1-\hat{z})\right)\right]+\tilde{G}_{F}\left(x, x_{B}\right) \frac{1}{\varepsilon} \delta(1-\hat{z})\right\}+\cdots
\end{aligned}
$$

The last term associated with another twist-3 function $\tilde{G}_{F}\left(x, x_{B}\right)$ was found in [12] and other part was calculated in [8].
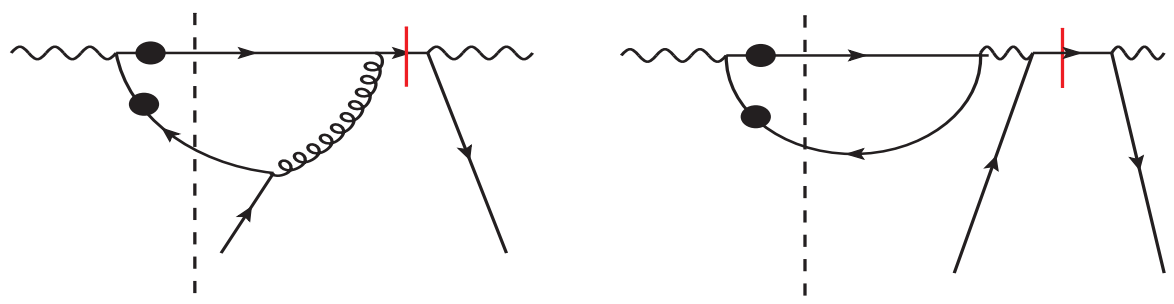

Figure 4: Diagrammatic description for HP2 contribution.

Finally we discuss the HP2 contribution shown in Fig.4. This was first calculated in [12] as

$$
\begin{aligned}
& \frac{d^{4}\left\langle P_{h \perp} \Delta \sigma\right\rangle^{\mathrm{HP} 2}}{d x_{B} d Q^{2} d z_{h} d \phi} \\
= & -\frac{\pi M_{N} \alpha_{e m}^{2}}{4 x_{B}^{2} S_{e p}^{2} Q^{2}} \frac{\alpha_{s}}{2 \pi}\left(\frac{4 \pi \mu^{2}}{Q^{2}}\right)^{\varepsilon} \frac{1}{\Gamma(1-\varepsilon)} \int d z D(z) \int \frac{d x}{x} \\
& \times \frac{1}{2 N}\left\{-G_{F}\left(x_{B}, x_{B}-x\right) \frac{1}{\varepsilon}(1-2 \hat{x}) \delta(1-\hat{z})-\tilde{G}_{F}\left(x_{B}, x_{B}-x\right) \frac{1}{\varepsilon} \delta(1-\hat{z})\right\}+\cdots
\end{aligned}
$$




\section{Scale evolution equation of $G_{F}(x, x)$}

After combining all contributions in previous sections, we can obtain the NLO $P_{h \perp}$-weighted cross section as

$$
\begin{aligned}
& \frac{d^{4}\left\langle P_{h \perp} \Delta \sigma\right\rangle^{\mathrm{LO}+\mathrm{NLO}}}{d x_{B} d Q^{2} d z_{h} d \phi}=-\frac{z_{h} \pi M_{N} \alpha_{e m}^{2}}{4 x_{B}^{2} S_{e p}^{2} Q^{2}} \frac{\alpha_{s}}{2 \pi}\left(\frac{4 \pi \mu^{2}}{Q^{2}}\right)^{\varepsilon} \frac{1}{\Gamma(1-\varepsilon)} \sum_{q} e_{q}^{2} \\
& \times\left[( - \frac { 1 } { \varepsilon } ) \left\{D ^ { q } ( z _ { h } ) \left\{\int _ { x _ { B } } ^ { 1 } \frac { d x } { x } \left[C_{F} P_{q q}(\hat{x}) G_{F}^{q}(x, x)+\frac{N}{2}\left(\frac{(1+\hat{x}) G_{F}^{q}\left(x_{B}, x\right)-\left(1+\hat{x}^{2}\right) G_{F}^{q}(x, x)}{(1-\hat{x})_{+}}\right.\right.\right.\right.\right. \\
& \left.\left.\left.+\tilde{G}_{F}^{q}\left(x_{B}, x\right)\right)\right]-N G_{F}^{q}\left(x_{B}, x_{B}\right)+\frac{1}{2 N} \int_{x_{B}}^{1} \frac{d x}{x}\left((1-2 \hat{x}) G_{F}^{q}\left(x_{B}, x_{B}-x\right)+\tilde{G}_{F}^{q}\left(x_{B}, x_{B}-x\right)\right)\right\} \\
& \left.\left.+G_{F}^{q}\left(x_{B}, x_{B}\right) C_{F} \int_{z_{h}}^{1} \frac{d z}{z} P_{q q}(\hat{z}) D^{q}(z)\right\}\right]+\cdots,
\end{aligned}
$$

where $P_{q q}(x)$ is the splitting function

$$
P_{q q}(x)=C_{F}\left[\frac{1+x^{2}}{(1-x)_{+}}+\frac{3}{2} \delta(1-x)\right] .
$$

From the structure of the collinear divergence $1 / \varepsilon$, we can derive the scale evolution equation of $G_{F}(x, x)$ as

$$
\begin{aligned}
& \frac{\partial}{\partial \ln \mu^{2}} G_{F}\left(x_{B}, x_{B}, \mu^{2}\right)=\frac{\alpha_{s}}{2 \pi}\left\{\int _ { x _ { B } } ^ { 1 } \frac { d x } { x } \left[P_{q q}(\hat{x}) G_{F}\left(x, x, \mu^{2}\right)\right.\right. \\
& \left.+\frac{N}{2}\left(\frac{(1+\hat{x}) G_{F}\left(x_{B}, x, \mu^{2}\right)-\left(1+\hat{x}^{2}\right) G_{F}\left(x, x, \mu^{2}\right)}{(1-\hat{x})_{+}}+\tilde{G}_{F}\left(x_{B}, x, \mu^{2}\right)\right)\right]-N G_{F}\left(x_{B}, x_{B}, \mu^{2}\right) \\
& \left.+\frac{1}{2 N} \int_{x_{B}}^{1} \frac{d x}{x}\left((1-2 \hat{x}) G_{F}\left(x_{B}, x_{B}-x, \mu^{2}\right)+\tilde{G}_{F}\left(x_{B}, x_{B}-x, \mu^{2}\right)\right)\right\},
\end{aligned}
$$

which completely agrees with the results in other approaches $[4,6,7]$.

\section{References}

[1] Z. B. Kang and J. W. Qiu, Phys. Rev. D 79, 016003 (2009).

[2] J. Zhou, F. Yuan and Z. T. Liang, Phys. Rev. D 79, 114022 (2009).

[3] W. Vogelsang and F. Yuan, Phys. Rev. D 79, 094010 (2009).

[4] V. M. Braun, A. N. Manashov and B. Pirnay, Phys. Rev. D 80, 114002 (2009).

[5] A. Schafer and J. Zhou, Phys. Rev. D 85, 117501 (2012).

[6] J. P. Ma and Q. Wang, Phys. Lett. B 715, 157 (2012).

[7] Z. B. Kang and J. W. Qiu, Phys. Lett. B 713, 273 (2012).

[8] Z. B. Kang, I. Vitev and H. Xing, Phys. Rev. D 87, 034024 (2013).

[9] H. Eguchi, Y. Koike and K. Tanaka, Nucl. Phys. B 763, 198-227 (2007).

[10] Y. Koike and K. Tanaka, arXiv:0907.2797 [hep-ph].

[11] Y. Koike W. Vogelsang and F. Yuan, Phys. Lett. B 659, 878 (2008).

[12] S. Yoshida, Phys. Rev. D 93, 054048 (2016). 